\title{
Excitation of kinetic Alfvén turbulence by MHD waves and energization of space plasmas
}

\author{
Y. Voitenko ${ }^{1, *}$ and M. Goossens ${ }^{1}$ \\ ${ }^{1}$ Centre for Plasma Astrophysics, K. U. Leuven, Belgium \\ * On leave from: Main Astronomical Observatory, Kyiv, Ukraine
}

Received: 19 September 2004 - Revised: 5 November 2004 - Accepted: 9 November 2004 - Published: 16 November 2004

Part of Special Issue "Advances in space environment turbulence"

\begin{abstract}
There is abundant observational evidence that the energization of plasma particles in space is correlated with an enhanced activity of large-scale MHD waves. Since these waves cannot interact with particles, we need to find ways for these MHD waves to transport energy in the dissipation range formed by small-scale or high-frequency waves, which are able to interact with particles. In this paper we consider the dissipation range formed by the kinetic Alfvén waves (KAWs) which are very short- wavelengths across the magnetic field irrespectively of their frequency. We study a nonlocal nonlinear mechanism for the excitation of KAWs by MHD waves via resonant decay $A W(F W) \rightarrow$ $K A W_{1}+K A W_{2}$, where the MHD wave can be either an Alfvén wave (AW), or a fast magneto-acoustic wave (FW). The resonant decay thus provides a non-local energy transport from large scales directly in the dissipation range. The decay is efficient at low amplitudes of the magnetic field in the MHD waves, $B / B_{0} \sim 10^{-2}$. In turn, KAWs are very efficient in the energy exchange with plasma particles, providing plasma heating and acceleration in a variety of space plasmas. An anisotropic energy deposition in the field-aligned degree of freedom for the electrons, and in the cross-field degrees of freedom for the ions, is typical for KAWs. A few relevant examples are discussed concerning nonlinear excitation of KAWs by the MHD wave flux and consequent plasma energization in the solar corona and terrestrial magnetosphere.
\end{abstract}

\section{Introduction}

In-situ and remote observations show the presence of MHD waves in a variety of space plasmas - for example the solar corona, the solar wind, and planetary magnetospheres. These waves can be excited by different drivers, like MHD plasma instabilities and mechanical plasma motions (Verheest, 1977;

Correspondence to: Y. Voitenko

(yuriy.voitenko@wis.kuleuven.ac.be)
Fushiki and Sakai, 1994). It is believed that the plasma energization that accompanies an enhanced activity of MHD waves, has its source in these waves. However, MHD waves are excited at length scales and time scales that are far longer than those required for efficient dissipation. Hence energy carried by MHD waves can only be available for the energization of the plasma if there exist mechanisms that can transport the energy from the long wavelengths at excitation to the required short wavelengths for dissipation.

This problem particularly shows up in recent SOHO observations of the solar corona. Spectroscopic SOHO observations of the widths of extreme ultraviolet emission lines indicate that ions are very hot in the high corona at 1.5-4 solar radii (Kohl et al., 1997). Moreover, the ion kinetic temperatures seem to be highly anisotropic with the perpendicular (with respect to the background magnetic field) temperature being much higher than the parallel temperature (Kohl et al., 1997; Dodero et al., 1998; Cranmer, 2002). These observations suggest that the ions are energized (heated and/or accelerated) anisotropically, mainly across the magnetic field. An attempt to explain the perpendicular energization of the ions in the solar corona as due to the ion-cyclotron heating by high-frequency waves (see recent papers by Marsch and Tu (2001), and by Hollweg and Isenberg (2002), and references therein) leads to questions about the origin of these high-frequency $\left(10^{2} \div 10^{4} \mathrm{~Hz}\right)$ waves at those large heights in the corona. If these waves are excited at the coronal base (Voitenko and Goossens, 2002a), then it is difficult to explain how they can propagate so far. At the same time, a strong anti-sunward flux of MHD waves should exist throughout the whole corona, and it is permanently observed in the solar wind. The source for this flux can be the convective motions at the photosphere, which can generate very lowfrequency waves, $f=10^{-4} \div 10^{-2} \mathrm{~Hz}$, or the magnetic activity (magnetic reconnection and restructuring) in the photosphere/chromosphere/low corona, which can generate waves in an intermediate-frequency band $f=10^{-1} \div 10^{0} \mathrm{~Hz}$. Having in mind the difficulties with the penetration of very lowfrequency waves from the photosphere into corona, it is of- 
ten suggested that just these intermediate-frequency waves provide the main energy source for the coronal heating and solar wind acceleration (Ryutova et al., 2001). Then one can envisage that the upward flux of MHD waves generates the ion-cyclotron waves in-situ. Direct turbulent cascade towards higher frequencies (Li et al., 1999), and generation by currents carried by MHD waves (Markovskii and Hollweg, 2002) have been proposed as local and non-local mechanisms to transport wave energy to the ion-cyclotron dissipation range.

However, under solar corona conditions the turbulent cascade is anisotropic and proceeds towards high cross-field wave numbers rather than to high field-aligned wave numbers which are required for ion-cyclotron resonance (Leamon et al., 2000; Matthaeus et al., 2003). Phase mixing in transversally nonuniform plasmas works in the same direction, but it appears to be too slow in the corona for providing the necessary rate of transfer of wave energy to the dissipative length scales, which are of the order of the ion (proton) gyroradius $\rho_{p}$. In addition, the turbulent cascade can develop only under restricting special conditions, like the presence of a fraction of counter-propagating waves. While a partial reflection of the very low-frequency $\left(<10^{-2} \mathrm{~Hz}\right)$ Alfvén waves in a vertically stratified corona can give rise to the counter-propagating waves (Leamon et al., 2000; Matthaeus et al., 2003), for the intermediate-frequency waves, $f=$ $10^{-1} \div 10^{0} \mathrm{~Hz}$ (actually, for $f>10^{-2} \mathrm{~Hz}$ ), the reflection is negligible. The nonlocal transport due to current instabilities (Markovskii and Hollweg, 2002), can only be efficient when MHD waves develop strong shear flows or currents. Hence, this type of transport requires pre-structured Alfvén waves with sufficiently small scales either across the background magnetic field, or in the field-aligned direction (which in turn implies already high-frequency Alfvén waves).

In the present paper we study nonlinear processes that can strongly accelerate the evolution of wave energy towards small length-scales: the resonant three-wave decays of large-scale MHD Alfvén and fast waves into kinetic Alfvén waves, $A W(F W) \rightarrow K A W_{1}+K A W_{2}$. In the framework of two-fluid MHD we show that finite-amplitude MHD waves are nonlinearly coupled to kinetic Alfvén waves (KAWs). The kinetic Alfvén waves we refer to are Alfvén waves with short wavelengths across the background magnetic field $B_{0}$ (large perpendicular wavenumbers $k_{\perp} \gg k_{\|}$). Because of their ability to interact strongly with plasmas and with other wave modes, KAWs are now under intensive investigation (Stasiewicz et al., 2000; Voitenko and Goossens, 2002a; Onischenko et al., 2004). The kinetic and inertial regimes of KAWs have been observed in the laboratory (Leneman et al., 1999; Kletzing et al., 2003) and in space (Wygant et al., 2002; Chaston et al., 2003; Stasiewicz et al., 2004) plasmas. The non-local three-wave resonance of a large-scale MHD wave with small-scale daughter KAWs is possible because of the highly anisotropic nature of KAWs which are still lowfrequency despite of the high oblique wavenumbers. Important advantages of this nonlocal transport are that it is not restricted to any specific wavelengths or frequencies of initial
MHD waves, and that it does not require the pre-existence of counter-propagating waves or plasma non-uniformities.

For high oblique wavenumbers the electric field of nonlinearly driven KAWs has important components parallel to the equilibrium magnetic field, $\mathbf{E}_{\|} \| \mathbf{B}_{0}$, and parallel to the cross-field wave vector, $\mathbf{E}_{\perp} \| \mathbf{k}_{\perp}$. Because of the parallel electric field the KAWs undergo the Cherenkov resonance with the electrons, and heat and accelerate them along $\mathbf{B}_{0}$. This process can explain the high electron energy in the parallel (field-aligned) direction, observed in aurora (Wygant et al., 2002). On the other hand, the perpendicular electric field $\mathbf{E}_{\perp} \| \mathbf{k}_{\perp}$ leads to a strong cross-field acceleration of the ions which occurs in the vicinity of demagnetizing KAW phases (Voitenko and Goossens, 2004). This process can explain a large excess of ion energy in the cross-field plane, observed in the solar corona (Dodero et al., 1998) and in aurora (Chaston et al., 2003).

\section{Eigenmode KAW equation}

We consider a uniform hydrogen plasma immersed in a background magnetic field $B_{0}$. In terms of the effective density potential $\phi$,

$\phi=\frac{T_{e}}{e} \ln \frac{n_{e}}{n_{0}}$,

the nonlinear (up to second order in perturbation amplitudes) eigenmode equation for KAWs is (Voitenko and Goossens, 2002b):

$$
\left(\frac{\partial^{2}}{\partial t^{2}}-V_{A}^{2} K^{2} \nabla_{\|}^{2}-2 \gamma_{\mathrm{d}} \frac{\partial}{\partial t}\right) \phi=N_{\text {tot }} .
$$

$\gamma_{\mathrm{d}}$ is the linear damping/growth rate due to wave-particle interaction, $V_{A}=B_{0} / \sqrt{4 \pi n m_{p}}$ is the Alfvén velocity, $\nabla_{\|}$ is the spatial derivative along $\mathbf{B}_{0}$, and $K$ is the dispersion function so that the wave dispersion in wavenumber space is $\omega=k_{\|} V_{A} K$. In low- $\beta$ plasmas $K$ can be approximated as

$\frac{\omega^{2}}{k_{\|}^{2} V_{A}^{2}}=K^{2} \approx \frac{1+\mu_{T}}{1+\chi_{e}}$,

where $\mu_{T}=\rho_{T}^{2} k_{\perp}^{2}, \rho_{T}^{2}=V_{T}^{2} / \Omega_{p}^{2}, V_{T}^{2}=T / m_{p}, T=$ $T_{p}+T_{e}, \Omega_{p}=e B_{0} / m_{p} c$ is the ion-cyclotron frequency, $\beta=V_{T}^{2} / V_{A}^{2}$ is the gas/magnetic pressure ratio, $\chi_{e}=\delta_{e}^{2} k_{\perp}^{2}=$ $\beta^{-1}\left(m_{e} / m_{p}\right) \mu_{T}, \delta_{e}$ is the electron inertial length. The KAW dispersion function $K>1$ for $m_{e} / m_{p}<\beta<1$, and $K<1$ for $\beta<m_{e} / m_{p}$.

The nonlinear part $N_{\text {tot }}$ of Eq. (2) is quite complicated:

$$
\begin{aligned}
& \frac{1+\delta_{e}^{2} k_{\perp}^{2}}{V_{T e}^{2}} N_{\mathrm{tot}} \\
& =-V_{T e}^{-2}\left[\left[\frac{m_{e}}{m_{p}} \bar{\triangle}_{p}+\left(1-\bar{v}_{k}^{-2}\right)\right] \frac{\partial}{\partial t} \mathbf{v}_{e} \cdot \nabla+\frac{\partial}{\partial t} \nabla_{\|}\right. \\
& \left.\left(v_{e \|}-v_{p \|}\right)+\bar{v}_{k}^{-2} \frac{\partial}{\partial t}\left(\mathbf{v}_{p}-\mathbf{v}_{e}\right) \cdot \nabla\right] \phi
\end{aligned}
$$


$+\left[\frac{m_{e}}{m_{p}} \bar{\triangle}_{p}\right] \nabla_{\|} \cdot \mathbf{F}_{e \|}+\frac{m_{e}}{m_{p}}\left[\frac{m_{e}}{m_{p}} \bar{\triangle}_{p}+\frac{m_{e}}{m_{p}}\left[1-\bar{v}_{k}^{-2}\right]\right]$

$\frac{1}{\Omega} \frac{\partial}{\partial t} \nabla_{\perp} \cdot\left[\mathbf{b}_{0} \times \mathbf{F}_{e \perp}\right]$

$+\frac{m_{e}}{m_{p}}\left(1-\bar{v}_{k}^{-2}\right) \nabla_{\|} \cdot\left(\mathbf{F}_{e \|}-\mathbf{F}_{p \|}\right)+\frac{m_{e}}{m_{p}} \bar{v}_{k}^{-2} \frac{1}{\Omega} \frac{\partial}{\partial t} \nabla_{\perp}$.

$\left[\mathbf{b}_{0} \times\left(\mathbf{F}_{e \perp}-\mathbf{F}_{p \perp}\right)\right]+\frac{m_{e}}{m_{p}} \bar{v}_{k}^{-2} \frac{1}{\Omega^{2}} \frac{\partial^{2}}{\partial t^{2}} \nabla_{\perp} \cdot \mathbf{F}_{p \perp}$.

The nonlinear second-order terms come from the electron and ion continuity equations, and from the parallel and perpendicular components of the Ampére law. The nonlinear force

$\mathbf{F}_{s}=\frac{1}{c} \mathbf{v}_{s} \times \mathbf{B}-\frac{m_{s}}{q_{s}}\left(\mathbf{v}_{s} \cdot \nabla\right) \mathbf{v}_{s}$.

The relative number density fluctuations with respect to magnetic fluctuations in KAWs is

$\frac{n}{n_{0}}=-i k_{\perp} \delta_{p} \frac{K}{K^{2}-\beta} \frac{B_{\perp}}{B_{0}}$.

The magnetic compressibility is

$\frac{B_{\|}}{B_{0}}=-\beta \frac{n}{n_{0}}$.

This shows that the gas compression is more important for AWs than the magnetic compression in a low- $\beta$ plasma. In terms of $\phi_{k}$, we have the following expressions for the perturbed velocity and magnetic field due to KAWs:

$\mathbf{v}_{p \perp}=\frac{c}{B_{0}} \mu_{T e}^{-1}\left[\frac{1}{\Omega_{p}} \frac{\partial}{\partial t}(1-\beta) \nabla_{\perp} \phi-\mathbf{b}_{0} \times \nabla_{\perp} \phi_{k}\right] ;$

$\mathbf{v}_{e \perp}=\frac{c}{B_{0}} \mu_{T e}^{-1}\left[-\beta \frac{1}{\Omega_{p}} \frac{\partial}{\partial t} \nabla_{\perp}-\left(1+\mu_{T}\right) \mathbf{b}_{0} \times \nabla_{\perp}\right] \phi_{k} ;$

$v_{e z}=s \frac{e}{m_{e}} \frac{V_{A}}{V_{T e}^{2}} K \phi_{k}$;

$v_{p z}=-s \frac{e}{m_{e}} \frac{V_{A}}{V_{T e}^{2}} \frac{m_{e} / m_{p}-\beta}{1+\mu_{T}} K \phi_{k} ;$

$\mathbf{B}_{\perp}=s \frac{c}{V_{A}} \frac{K}{\mu_{T e}}\left[\mathbf{b}_{0} \times \nabla_{\perp} \phi_{k}\right]$,

where $\mu_{T e}=\mu_{T} T_{e} /\left(T_{e}+T_{p}\right)$, and $s \equiv \operatorname{sign}\left(k_{z}\right)$ indicates the direction of KAW's propagation.

\section{Nonlinear resonant excitation of KAWs by MHD waves}

\subsection{Resonant decay}

We concentrate here on the processes induced by the scalar nonlinearities (Voitenko and Goossens, 2002b): resonant decays of the large-scale pump Alfvén and fast magnetoacoustic waves into KAWs. These processes result in a jump-like spectral transport of MHD wave energy directly into the dissipation range (i.e., dissipative length scales). We consider the most efficient three-wave resonant interactions (Sagdeev and Galeev, 1969). The beatings between "pump" wave with wave vector $\mathbf{k}_{\mathrm{P}}$ and trial KAW $\mathbf{k}_{1}$ can efficiently drive the complimentary trial KAW $\mathbf{k}_{2}$ with spatio-temporal scales that coincide with the scales of the beatings: $\mathbf{k}_{2}=\mathbf{k}_{\mathrm{P}} \pm \mathbf{k}_{1}$; $\omega\left(\mathbf{k}_{2}\right)=\omega_{\mathrm{P}}\left(\mathbf{k}_{\mathrm{P}}\right) \pm \omega\left(\mathbf{k}_{1}\right)$. If there is initially a low (e.g., thermal) level of KAWs, the efficient amplification of KAWs occurs when there is a positive back reaction on the wave $\mathbf{k}_{1}$ from the wave $\mathbf{k}_{2}$, i.e. when the beatings between the KAW $\mathbf{k}_{2}$ and the pump $\mathbf{k}_{\mathrm{P}}$ in turn drive $\mathbf{k}_{1}: \mathbf{k}_{1}=\mathbf{k}_{\mathrm{P}} \pm \mathbf{k}_{2}$; $\omega\left(\mathbf{k}_{1}\right)=\omega_{\mathrm{P}}\left(\mathbf{k}_{\mathrm{P}}\right) \pm \omega\left(\mathbf{k}_{2}\right)$. In this case the process takes the form of (parametric) decay, where the pump wave decays into a spectrum of wave pairs which satisfy the resonant conditions for wave vectors

$\mathbf{k}_{1}+\mathbf{k}_{2}=\mathbf{k}_{\mathrm{P}}$,

and wave frequencies

$\omega\left(\mathbf{k}_{1}\right)+\omega\left(\mathbf{k}_{2}\right)=\omega_{\mathrm{P}}\left(\mathbf{k}_{\mathrm{P}}\right)$.

Vectorial nonlinear interaction of KAWs has been studied by (Voitenko, 1998a) (kinetic theory) and (Voitenko and Goossens, 2000, 2002b) (2-fluid resistive MHD). This interaction is proportional to $\mathbf{k}_{1 \perp} \times \mathbf{k}_{2 \perp}$ and is therefore local in $k$-space, $\left|\mathbf{k}_{1 \perp}\right| \sim\left|\mathbf{k}_{2 \perp}\right| \sim\left|\mathbf{k}_{\mathrm{P} \perp}\right|$. Scalar interaction is formally of order $\beta$, but since it is proportional to $\mathbf{k}_{1 \perp} \cdot \mathbf{k}_{2 \perp}$, the resonant non-local interaction is possible where $\left|\mathbf{k}_{1 \perp}\right| \sim\left|\mathbf{k}_{2 \perp}\right| \gg\left|\mathbf{k}_{\mathrm{P} \perp}\right|$. Indeed, from the resonant condition $\mathbf{k}_{\mathrm{P} \perp}=\mathbf{k}_{1 \perp}+\mathbf{k}_{2 \perp}$, we have $\mathbf{k}_{1 \perp} \sim-\mathbf{k}_{2 \perp}$ and then $\mathbf{k}_{1 \perp} \cdot \mathbf{k}_{2 \perp} \gg\left|\mathbf{k}_{1 \perp} \times \mathbf{k}_{2 \perp}\right|$ for the non-local interaction.

It is useful to factorize the KAW wave functions $\phi_{1}$ and $\phi_{2}$ into an exponential phase dependence and a slowly varying amplitude, $\Phi_{1,2}=\Phi_{1,2}(t)$ :

$\phi_{1,2}=\Phi_{1,2} \exp \left(-i \omega_{1,2} t+i \mathbf{k}_{1,2} \cdot \mathbf{r}\right)$.

The equations for resonant short-scale Alfvén waves 1 and 2 , coupled to the large-scale pump wave $\mathrm{P}$, are then obtained from the nonlinear eigenmode Eq. (2) as

$\left[\frac{\partial}{\partial t}-\gamma_{\mathrm{d} 1}\right] \Phi_{1}=U_{1,-2, \mathrm{P}} \Phi_{2}^{*} b_{\mathrm{P}}$

$\left[\frac{\partial}{\partial t}-\gamma_{\mathrm{d} 2}\right] \Phi_{2}^{*}=U_{-2,1,-\mathrm{P}} \Phi_{1} b_{\mathrm{P}}^{*}$,

$b_{\mathrm{P}}=B_{\mathrm{P}} / B_{0}$ is the normalized magnetic field of the pump wave. The coupling coefficients are

$U_{1,-2, \mathrm{P}}=\left(-i \omega_{1} \Phi_{2}^{*} b_{\mathrm{P}}\right)^{-1} N_{\text {tot }}(1 ;-2 ; \mathrm{P})$,

$U_{-2,1,-\mathrm{P}}=\left(i \omega_{2} \Phi_{1} b_{\mathrm{P}}^{*}\right)^{-1} N_{\text {tot }}(-2 ; 1 ;-\mathrm{P})$,

where $N_{\text {tot }}$ have to be calculated from Eq. (4) by eliminating all the pump variables in favor of $b_{\mathrm{P}}$, and by eliminating all 
the KAW variables in favor of $\Phi$. The total growth (or damping) rate of exponentially growing (or decaying) solutions of the system (Eqs. 14-15), $\Phi_{1,2} \sim \exp \left(\gamma_{\text {tot }} t\right)$, is

$$
\gamma_{\mathrm{tot}}=\frac{\gamma_{\mathrm{d} 1}+\gamma_{\mathrm{d} 2}}{2} \pm \sqrt{\left(\frac{\gamma_{\mathrm{d} 1}-\gamma_{\mathrm{d} 2}}{2}\right)^{2}+\gamma_{N L}^{2}}
$$

where $\gamma_{N L}$ is the nonlinear growth rate - the rate of the nonlinear pumping of MHD wave energy into daughter KAWs:

$\gamma_{N L}=\sqrt{U_{1,-2, \mathrm{P}} U_{-2,1,-\mathrm{P}}}\left|b_{\mathrm{P}}\right|$.

3.2 Nonlinear decay of fast waves into kinetic Alfvén waves

Let us consider a pump FW with a frequency $\omega_{\mathrm{P}}$ propagating at an angle to the background magnetic field, $\mathbf{k}_{\mathrm{P}}=\left(k_{\mathrm{P} \perp} ; 0 ; k_{\mathrm{P} z}\right)$. The $z$-axis is parallel to $\mathbf{B}_{0}$, and we assume that $k_{\mathrm{P} z}>0$. To derive the nonlinear second-order parts of the equations for the KAWs 1 and 2, we use the linear relation for KAWs given in the previous section and the standard linear relation for the pump FW:

$\phi_{\mathrm{P}}=\frac{m_{e} V_{T e}^{2}}{e} K_{\mathrm{P}} \frac{B_{\mathrm{P} \|}}{B_{0}} \equiv \frac{m_{e} V_{T e}^{2}}{e} K_{\mathrm{P}} b_{\mathrm{P} \|}$,

(in terms of number density this means $n_{\mathrm{P}} / n_{0}=$ $\left.K_{\mathrm{P}} B_{\mathrm{P} \|} / B_{0}\right)$. The perpendicular electron and ion velocity in the pump FW are equal, $\mathrm{V}_{\mathrm{P} e \perp}=\mathrm{V}_{\mathrm{P} i \perp} \equiv \mathrm{V}_{\mathrm{P} \perp}$,

$\mathbf{V}_{\mathrm{P} \perp}=\mathbf{e}_{\mathrm{P} \perp} \frac{\omega_{\mathrm{P}}}{k_{\mathrm{P} \perp}} b_{\mathrm{P} \|}$,

and the magnetic field components are related through $\nabla \cdot \mathbf{B}=\mathbf{0}$ as

$\mathbf{B}_{\mathrm{P} \perp}=-\mathbf{e}_{\mathrm{P} \perp} \frac{k_{\mathrm{P} z}}{k_{\mathrm{P} \perp}} B_{\mathrm{P} \|}$.

The function $K_{\mathrm{P}}$ accounts for the thermal correction in the oblique fast waves:

$K_{\mathrm{P}}=\frac{\omega_{\mathrm{P}}}{k_{\mathrm{P}} V_{A}}=\sqrt{1+\beta \frac{k_{\mathrm{P} \perp}^{2}}{k_{\mathrm{P}}^{2}}}$

As we are interested in a non-local process of energy transport into a region of high perpendicular wavenumbers, we consider excited waves with high perpendicular wavenumbers, $\left|k_{1 \perp}\right| \approx\left|k_{2 \perp}\right| \gg k_{\mathrm{P} \perp}$. In that case we have $-\mathbf{k}_{1 \perp} \approx$ $\mathbf{k}_{2 \perp} \equiv \mathbf{k}_{\perp}$ (dipole approximation in the perpendicular direction) and $K\left(k_{2 \perp}\right) \approx K\left(k_{1 \perp}\right) \equiv K$. Assuming $s_{1}=1$, the frequency matching condition,

$\omega_{1}+\omega_{2}-\omega_{\mathrm{P}}=0$.

and the parallel wavenumber matching condition,

$k_{1 z}+s_{2}\left|k_{2 z}\right|=k_{\mathrm{Pz}}$,

can be solved for $s_{2}=-1$ (anti-parallel propagation of two product KAWs along the background magnetic field, $\mathbf{k}_{1 z}$. $\mathbf{k}_{2 z}<0$ ). In this case the frequencies of decay waves for given $K=K\left(k_{\perp}\right)$ are

$\omega_{1}=\frac{1}{2}\left(1+\frac{k_{\mathrm{P} z}}{k_{\mathrm{P}}} \frac{K}{K_{\mathrm{P}}}\right) \omega_{\mathrm{P}}$;

$\omega_{2}=\frac{1}{2}\left(1-\frac{k_{\mathrm{P} z}}{k_{\mathrm{P}}} \frac{K}{K_{\mathrm{P}}}\right) \omega_{\mathrm{P}}$.

For $s_{2}=1$ (parallel propagation of two product KAWs along the background magnetic field), we find from Eq. (22) that the KAWs' wavenumbers should be fixed, $\mu_{T}=\mu_{T}^{*} \equiv$ $(1+\beta) k_{\mathrm{P} \perp}^{2} / k_{\mathrm{P} z}^{2}$. The decay of fast waves into parallel propagating KAWs is thus selective (restricted by the matching conditions). For $s_{2}=-1$ (anti-parallel propagation), the matching conditions reduce to the requirement $\omega_{2}>0$, which can be satisfied in a wide range of perpendicular wavenumbers $\mu_{T}<\mu_{T}^{*}$.

The nonlinear coupling coefficients for the waves 1 and 2, $U_{1,-2, \mathrm{P}}=N_{1} k_{\mathrm{P} \perp} / k_{\mathrm{P}}, U_{-2,1,-\mathrm{P}}=N_{2} k_{\mathrm{P} \perp} / k_{\mathrm{P}}$,

$N_{1,2}=-K_{\mathrm{P}} \frac{\omega_{1,2}}{\omega_{\mathrm{P}}}-\frac{1}{K^{2}} \frac{2 \omega_{2,1}}{\omega_{\mathrm{P}}}+s_{1,2} \frac{K}{K_{\mathrm{P}}} \frac{k_{\mathrm{P} z}}{k_{\mathrm{P}}}+\frac{\mathbf{k}_{\mathrm{P} \perp} \cdot \mathbf{k}_{1,2 \perp}}{k_{\mathrm{P} \perp}^{2}} \times$

$\left(\left(1-K_{\mathrm{P}}\right)\left(1-s_{1,2} \frac{K}{K_{\mathrm{P}}} \frac{k_{\mathrm{P} z}}{k_{\mathrm{P}}}\right)+\beta\left(1-\frac{1}{K^{2}}\right)^{2}+\frac{\beta}{K^{2}}\right)$,

follow from Eq. (4) where all the KAW perturbations are eliminated in terms of $\phi_{1,2}$, and all the fast wave perturbations are eliminated in terms of $b_{\mathrm{P}}$.

The threshold amplitude of the decay in the collisional regime, where the wave damping is determined by collisional dissipation $\gamma_{\mathrm{d} 1}=\gamma_{\mathrm{d} 2}=\gamma_{\mathrm{d}}($ Eq. 47), is

$b_{\mathrm{P}}^{C} \approx 10^{-2} \frac{v_{e}}{\omega_{\mathrm{P}}}$.

The collisional threshold for a pump frequency as low as $\omega_{\mathrm{P}} \sim 0.1 v_{e}$ is $\left|b_{\mathrm{P}}^{C}\right| \sim 0.1$. For higher frequencies the threshold decreases to values $\left|b_{\mathrm{P}}^{C}\right| \lesssim 10^{-2}$ at $\omega_{\mathrm{P}} \sim v_{e}$.

At higher pump frequencies, $\omega_{\mathrm{P}}>v_{e}$, the instability switches into the collisionless regime where the Landau damping of KAWs dominates over the collisional damping. The threshold amplitude of the decay in the collisionless regime is

$b_{\mathrm{P}}^{L}=\frac{\mu_{T}}{K} \sqrt{\frac{\pi}{4} \frac{m_{e}}{\beta m_{p}} \frac{1}{N_{1} N_{2}\left(1-\cos ^{2} \theta_{\mathrm{P}}\right)}\left(1-\cos ^{2} \theta_{\mathrm{P}} \frac{K^{2}}{K_{\mathrm{P}}^{2}}\right)}$.

$b_{\mathrm{P}}^{L}$ is a decreasing function of $\mu_{T}$ and attains its minimum $b_{\mathrm{thr}}^{L}$ at the maximal possible value of $\mu_{T} \lesssim \mu_{T}^{*}$. The values of this minimum determine the instability threshold as a function of $\cos ^{2} \theta_{\mathrm{P}}$. In the limit $\mu_{T} \rightarrow \mu_{T}^{*}$ we obtain $b_{\mathrm{P}}^{L} \rightarrow 0$, and there is no any threshold for the instability. But, as follows from Eq. (25), in the limit $\mu_{T} \rightarrow \mu_{T}^{*}$ we have $\omega_{1} \rightarrow 0$, that is $\lambda_{1 z} \rightarrow \infty$, which is prohibited by the finite parallel length scale of inhomogeneity in real systems. The real threshold has then to be calculated in a different limit $\mu_{T} \rightarrow \mu_{\max }\left(\mu_{\max }<\mu_{T}^{*}\right)$, where $\mu_{\max }$ is determined by the maximal parallel wavelength allowed by the parallel system size $L_{z}: \lambda_{1 z}\left(\mu_{\max }\right)=2 \pi V_{A} K\left(\mu_{\max }\right)=L_{z}$. 
3.3 Nonlinear decay of MHD Alfvén waves into kinetic Alfvén waves

We consider a pump AW with a frequency $\omega_{\mathrm{P}}$ and wave vector $\mathbf{k}_{\mathrm{P}}=\left(0 ; 0 ; k_{\mathrm{P} z}\right)$, that decays into KAWs with $k_{1 \perp} \approx$ $-k_{2 \perp} \gg k_{\mathrm{P} \perp}$ and with approximately equal dispersions, $K\left(k_{1 \perp}^{2}\right) \approx K\left(k_{2 \perp}^{2}\right)$. In that case $N_{\text {tot }}$ simplifies considerably because only the cross-field electromagnetic perturbations and velocity perturbations are induced by MHD Alfvén waves:

$\mathbf{v}_{\mathrm{P} p}=-s_{\mathrm{P}} V_{A}\left[\mathbf{b}_{\mathrm{P}}-i \frac{\omega_{\mathrm{P}}}{\Omega_{p}} \mathbf{b}_{0} \times \mathbf{b}_{\mathrm{P} \perp}\right]$

$\mathbf{v}_{\mathrm{P} e}=-s_{\mathrm{P}} V_{A} \mathbf{b}_{\mathrm{P} \perp}$

$B_{\mathrm{P} z}=v_{\mathrm{P} p z}=v_{\mathrm{P} e z}=0$.

The frequency matching condition, $\omega_{1}+\omega_{2}=\omega_{\mathrm{P}}$, and the parallel wavenumber matching condition, $s_{1} \omega_{1}+s_{2} \omega_{2}=$ $\omega_{\mathrm{P}} K$, can be satisfied only for antiparallel propagation of daughter KAWs (we take $s_{\mathrm{P}}=k_{\mathrm{P} z} /\left|k_{\mathrm{P} z}\right|$ ). So, for $s_{1}=1$, we must have $s_{2}=-1$. This determines the KAW frequencies as

$\omega_{1}=\frac{1+K}{2} \omega_{\mathrm{P}}$

and

$\omega_{2}=\frac{1-K}{2} \omega_{\mathrm{P}}$

The process is thus possible only if $K<1$, i.e., in a very low$\beta$ plasma with $\beta<m_{e} / m_{p}$. The KAWs in a $\beta<m_{e} / m_{p}$ plasma are sometimes called inertial Alfvén waves, because their dispersion is determined mainly by the parallel electron inertia. In these circumstances we obtain the following expressions for the electron and ion ponderomotive forces induced by the beatings of the pump Alfvén wave and the KAW with $\mathbf{b}_{\mathrm{P}} \times \nabla_{\perp} \phi_{k} \ll \mathbf{b}_{\mathrm{P}} \cdot \nabla_{\perp} \phi_{k}$ :

$\mu_{T e} \mathbf{F}_{e}=K^{2}\left(1-s_{\mathrm{P}} s \frac{1}{K}\right)\left(\mathbf{b}_{\mathrm{P}} \cdot \nabla_{\perp}\right) \phi_{k} \mathbf{b}_{0}$

$+s K \frac{k_{\perp}^{2} V_{A}}{\Omega_{p}} \phi_{k}\left[\mathbf{b}_{0} \times \mathbf{b}_{\mathrm{P}}\right]$

$\mu_{T e} \mathbf{F}_{p}=\left(1-s_{\mathrm{P}} s \frac{1}{K}\right)\left(\mathbf{b}_{\mathrm{P}} \cdot \nabla_{\perp}\right) \phi_{k} \mathbf{b}_{0}-\frac{V_{A}}{\Omega_{p}} s_{\mathrm{P}}\left(\mathbf{b}_{\mathrm{P}} \cdot \nabla_{\perp}\right)$

$\left[\mathbf{b}_{0} \times \nabla_{\perp}\right] \phi_{k}+\frac{V_{A}}{\Omega_{p}} s_{\mathrm{P}}\left(\mathbf{b}_{\mathrm{P}} \cdot \nabla_{\perp}\right) \frac{1}{\Omega_{p}} \frac{\partial}{\partial t} \nabla_{\perp} \phi_{k}$.

The coupling coefficients of KAWs with a pump MHD Alfvén wave are found as

$U_{1,-2, \mathrm{P}}=i s_{1} \frac{1}{K}\left(\frac{m_{p}}{m_{e}} \beta-K^{2}\right) \frac{\delta_{e}^{2} k_{\perp}^{2}}{1+\delta_{e}^{2} k_{\perp}^{2}} V_{A}\left(\mathbf{e}_{\mathrm{P}} \cdot \mathbf{k}_{2 \perp}\right) ;$

$U_{-2,1,-\mathrm{P}}=-i s_{2} \frac{1}{K}\left(\frac{m_{p}}{m_{e}} \beta-K^{2}\right) \frac{\delta_{e}^{2} k_{\perp}^{2}}{1+\delta_{e}^{2} k_{\perp}^{2}} V_{A}\left(\mathbf{e}_{\mathrm{P}} \cdot \mathbf{k}_{1 \perp}\right)$.
$\mathbf{e}_{\mathrm{P}}$ is the unit vector of the magnetic field of the pump wave, $\mathbf{e}_{\mathrm{P}}=\mathbf{B}_{\mathrm{P}} / B_{\mathrm{P}}$. The total growth rate of KAWs is given by Eq. (16), where the nonlinear pumping rate is found from Eq. (17) as

$\gamma_{N L}=\sqrt{\frac{m_{p}}{m_{e}}}\left|\frac{m_{p}}{m_{e}} \beta-1\right| \frac{\delta_{e}^{3} k_{\perp}^{3}}{\sqrt{1+\frac{m_{p}}{m_{e}} \beta \delta_{e}^{2} k_{\perp}^{2}}\left(1+\delta_{e}^{2} k_{\perp}^{2}\right)^{3 / 2}}\left|b_{\mathrm{P}}\right| \Omega_{p}$.

As long as $\beta$ is distinctly smaller than $m_{e} / m_{p}$, the MHD Alfvén waves decay very strongly, exciting KAWs. However, for $\beta \rightarrow m_{e} / m_{p}, \gamma_{N L} \rightarrow 0$. The decay is impossible for $\beta>m_{e} / m_{p}$, where the resonant conditions cannot be satisfied.

The strongest nonlinear pumping is in the KAWs with $k_{S \perp}^{2}=\left(1+\sqrt{1+3 / \beta_{m}}\right) \delta_{e}^{-2}$, with the (maximum) pumping rate

$\gamma_{N L \max }=\Omega_{p} \sqrt{\frac{3 m_{p}}{m_{e}}} \frac{\left(1-\beta_{m}\right)\left(\sqrt{\beta_{m}}+\sqrt{3+\beta_{m}}\right)}{\left(2 \sqrt{\beta_{m}}+\sqrt{3+\beta_{m}}\right)^{2}}\left|b_{\mathrm{P}}\right|$,

where $\beta_{m}=\beta m_{p} / m_{e}$.

These properties of the nonlinear growth rate, together with the dissipative properties of KAWs, create an interesting feedback loop for the plasma/MHD wave flux system. When the flux of MHD Alfvén waves enters the region where $\beta<m_{e} / m_{p}$, nonlinearly driven KAWs are generated and tend to heat the plasma up to the level where $\beta$ is kept slightly below $m_{e} / m_{p}, \beta=\beta_{c} \lesssim m_{e} / m_{p}$. When used in Eq. (38), this particular value $\beta_{c}$ provides a dynamical equilibrium, with the nonlinear growth rate $\gamma_{N L}$, at which the energy is pumped in the plasma via intermediate KAWs, balancing the rate of the energy loss from the plasma due to emission, thermal conduction and plasma acceleration. With different powers of the launched MHD Alfvén flux, the interplay of these processes can set up different regimes - from weak additional heating in the vicinity of the transition point $\beta=m_{e} / m_{p}$, to the creation of an extended region where $\beta \lesssim m_{e} / m_{p}$, which ends with accelerated flows of heated plasma, where $\beta>m_{e} / m_{p}$.

\section{Non-adiabatic cross-field acceleration of ions}

Nonlinearly driven KAWs have short wavelengths and are thus almost electrostatic in the sense that their electric field becomes almost parallel to the wave vector, $|\mathbf{k} \cdot \mathbf{E}| \gg$ $|\mathbf{k} \times \mathbf{E}|$. In this case the velocity of the polarization drift of ions becomes large, which makes KAWs available for the non-adiabatic ion acceleration and heating across the magnetic field (Voitenko and Goossens, 2004). Let us consider the motion of a initially thermal ion described by the equation of motion

$\frac{d}{d t} \mathbf{v}=\frac{q_{i}}{m_{i}} \mathbf{E}+\Omega_{i} \mathbf{v} \times \mathbf{e}_{z}+\Omega_{i} \mathbf{v} \times \frac{\mathbf{B}}{B_{0}}$,

where $\mathbf{e}_{z}$ is the unit vector in the direction of the mean magnetic field. Then, using the anisotropic properties of KAWs, 
we obtain the following equation for the perpendicular component of the ion velocity:

$\frac{d^{2}}{d t^{2}} v_{x}=\left[\frac{q_{i}}{m_{i}}\left(\frac{\partial E_{x}}{\partial x}-\frac{V_{i z}}{c} \frac{\partial B_{y}}{\partial x}\right)-\Omega_{i}^{2}\right] v_{x}$,

where the $x$-axis is taken in the direction of $E_{\perp}$, and we put $v_{z}=V_{i z}, V_{i z}$ is a characteristic velocity of the ions along the magnetic field, which is not strongly affected by the waves. Let us specify the magnetic field of the KAW as $B_{y}=$ $B_{y 0} \sin \left(\omega_{k} t-k_{z} z-k_{x} x\right)$. In the vicinity of the demagnetizing wave phases, $\omega_{k} t-k_{z} z(t)-k_{x} x(t) \approx \pi(2 l+1)(l$ integer), the ions can enter the regime of the exponential nonadiabatic acceleration,

$v_{x}=v_{x 0} \exp \left(\gamma_{\mathrm{n}-\mathrm{a}} t\right)$,

when the term in parentheses in Eq. (41) becomes positive. This condition determines the KAW parameters for the ion motion to lose its adiabatic character. Using the polarization relation for KAWs,

$E_{x}=\frac{V_{A}}{c} \frac{1+\mu_{p}}{K} B_{y}$,

we obtain the acceleration rate,

$\gamma_{\mathrm{n}-\mathrm{a}}=\Omega_{i} \sqrt{\frac{k_{x} V_{A}}{\Omega_{i}}\left(\frac{1+\mu_{p}}{K}-\frac{V_{i z}}{V_{A}}\right) \frac{B_{y 0}}{B_{0}}-1}$,

and the condition of super-adiabatic acceleration of ions $i$ in terms of magnetic KAW amplitude,

$\frac{k_{x} V_{A}}{\Omega_{i}}\left(\frac{1+\mu_{p}}{K}-\frac{V_{i z}}{V_{A}}\right) \frac{B_{y 0}}{B_{0}}>1$.

This condition determines the KAW parameters for the ion motion to lose its adiabatic character. In this case the crossfield velocity of the ions grows exponentially, which leads to the energization and heating of the ions. As long as $V_{i z} / V_{A} \ll 1$, the first term in the brackets is dominant (it is due to $\left.\partial E_{x} / \partial x\right)$. In the absence of waves, $B_{0 y}=0$, we have $\gamma_{\mathrm{n}-\mathrm{a}}= \pm i \Omega_{i}$, which corresponds to the usual cyclotron motion of ions.

It is clear from (43) that the differential field-aligned motion of the ions (second term in the brackets) can have a profound influence on their cross-field energization. Once the bulk velocity of given ions approaches the local Alfvén velocity, $V_{i z} \sim V_{A}$, their energization is suppressed. On the contrary, the energization is enforced for ions moving against the waves, $V_{i z} \sim-V_{A}<0$.

\section{Kinetic Alfvén turbulence}

Let us consider the excitation of KAW turbulence by the MHD Alfvén wave, which pumps energy into the mode $k_{S \perp} \sim \delta_{e}^{-1}$ (wave vector) at a rate $2 \gamma_{N L \text { max }}$. When the amplitude of a growing mode reaches its saturation value, parametric decay of the nonlinearly excited KAWs with $\mathbf{k}_{S}$ stops its growth, spreading out the wave spectrum. The decay into parallel-propagating KAWs initially spreads the spectrum into both higher and lower wavenumber domains, $k_{1 \perp}<k_{S \perp}<k_{2 \perp}$, whereas the decay only into counterstreaming KAWs spreads the spectrum into lower wavenumber domain, $k_{1 \perp}<k_{2 \perp}<k_{S \perp}$ (Voitenko, 1998a).

The relevance of the decay into counterstreaming waves for the problem of nonlinear saturation can be understood as follows. The time needed for the efficient energy exchange among counterstreaming KAWs, $\tau_{N L} \sim \gamma_{N L}^{-1}$ should be shorter than the overlapping time, $L_{c o r} / 2 V_{A}$, during which the counterstreaming wave travels in the field of the pump:

$\frac{L_{c o r}}{2 V_{A}} \gtrsim \gamma_{N L}^{-1}$.

Since the condition for saturation is $\gamma_{N L \max }^{-1}=\gamma_{k}^{-1}$, we obtain from (45) a quite reasonable estimate for the possible correlation length: $L_{c o r} \gtrsim 10 \lambda_{z}$, where $\lambda_{z}$ is the wavelength along $\mathbf{B}_{0}, \lambda_{z} \sim 10 \mathrm{~m}$.

The dynamics and direction of the nonlinear KAW energy transfer in $\mathbf{k}$-space have been investigated in a model of the triads interacting most effectively in the cascading process (Voitenko, 1998b). In this model the spectral energy cascade is considered as a series of consequent decays beginning with the 1-st step of the source wave decay into two secondary waves (decay products), having the largest growth rate. Any decay product of the $n$-th step in turn excites two decay products of the $(n+1)$-th step and so on, eventually resulting in the energy transfer in the $\mathbf{k}$-space, dynamical properties of which are determined by the form of matrix element of three-wave resonant interaction among KAWs $U\left(\mathbf{k}, \mathbf{k}_{1}, \mathbf{k}_{2}\right)$. The wavenumbers of the dominant decay products at any step are determined by maximizing $\left|U\left(\mathbf{k}_{1}, \mathbf{k},-\mathbf{k}_{2}\right) U\left(-\mathbf{k}_{2},-\mathbf{k}, \mathbf{k}_{1}\right)\right|$. In the case of the most effective decay into counterstreaming secondary waves, these wavenumbers are $k_{1 \perp} \approx 0.776 k_{s \perp}$ and $k_{2 \perp} \approx 0.442 k_{s \perp}$ in the limit of weak wave dispersion (we put $T_{e}=T_{i}$ for simplicity). Further pumping of energy into KAWs with $k_{S \perp}$ waves by MHD waves gives rise to further spreading of the wave spectrum and formation of the turbulent cascade.

In the KAW turbulence formed by dominant triads the energy tends to flow toward smaller $k$, forming a so-called inverse cascade. As the electron Landau damping and the collisional dissipation of KAWs are highly reduced at smaller $k_{\perp}$, this region can be considered as an inertial one (no sink no source), and an inertial-range power-law spectrum can be formed at $k_{\perp}<k_{s \perp}$. The strongest turbulent cascade including interaction among counterstreaming waves can form the inertial-range energy spectrum (Voitenko, 1998b):

$W_{k} \sim k_{z}^{-1 / 2} k_{\perp}^{-2}$.

This spectrum can exsist if there is a sink at some wavenumber $k_{\perp}^{*}, 0<k_{\perp}^{*}<k_{s \perp}$, othervise we encounter an infinite energy growth while $k_{\perp} \rightarrow 0$. If such a sink exists, the energy of the turbulence is concentrated at $k_{\perp} \gtrsim k_{\perp}^{*}$. It is possible that the non-adiabatic ion acceleration provides such sink, but in this case the inertial range can be quite short. 
An alternative spectral transport for KAWs induced by their nonlinear coupling to convective cells was considered by Pokhotelov et al. (2003) and Onischenko et al. (2004). Further investigations are required to decide which process and in what circumstances is more efficient for a finiteamplitude KAW: resonant decay into 2 counter-streaming KAWs, or decay into convective cells and KAW sidebands.

In the weak-turbulence limit shear-Alfvén waves propagating parallel to $B_{0}$ develop direct turbulent cascade and form an anisotropic energy spectrum that also scales as $k_{\perp}^{-2}$ (Bhattacharjee and $\mathrm{Ng}, 2001$ ).

\section{Some applications}

\subsection{Transient heating events in the solar atmosphere}

Transient brightenings on time scales of about one minute or shorter are observed in the low corona by Yohkoh and SOHO (blinkers, nano- and microflares) and attract an increasing interest (Berger et al., 1999; Berghmans et al., 2001). It is believed that most of these explosive events are closely related to the interaction of magnetic fluxes, separated by current sheets. Magnetic reconnection in current sheets may produce reconnection outflows and consequent plasma heating and line broadening due to plasma turbulence excited by the outflows, like in solar flares (Voitenko and Goossens, 2002a).

On the other hand, a considerable fraction of the energy can be released by the dynamical evolution of the current sheets themselves. Fushiki and Sakai (1994) have shown that fast waves can be produced in the solar atmosphere by a pinching current sheet. In turn, these fast waves undergo a modulational instability if the wave amplitudes overcome a critical value, $b_{\mathrm{P} \|}>V_{S} / V_{A}$ (Sakai, 1983; Fushiki and Sakai, 1994).

Let us consider the possibility that the fast waves with $\omega_{\mathrm{P}} \sim 10-100 \mathrm{~s}^{-1}$, emitted from the magnetic reconnection events, heat the surrounding plasma by the heavily damped KAWs that are excited by the resonant decay of the fast waves. For the coronal parameters the threshold amplitude (27) of the parametric decay is small, $b_{\mathrm{P} \|}^{C} \approx 10^{-2}$ for $\omega_{\mathrm{P}} \sim v_{e} \sim 10 \mathrm{~s}^{-1}$, and the resonant decay dominates under coronal conditions.

For the FW with an amplitude $b_{\mathrm{P}}=0.1$ and frequency $\omega_{\mathrm{P}}=v_{e}=30 \mathrm{~s}^{-1}$, pumped from the current sheet, we estimate the typical time for the decay instability to develop and the plasma to heat:

$\tau_{\text {tot }} \sim \tau_{N L} \sim \tau_{c} \sim 1 \mathrm{~s}$

These waves damp in the vicinity of the source (current sheet), and damp within the distance $L^{F W}=V_{A} \tau_{\text {tot }} \sim 10^{3}$ $\mathrm{km}$. Therefore the heated volume is $10^{3} \times 10^{3} \times L_{z} \mathrm{~km}^{3}\left(L_{z}\right.$ is the length of the current sheet, $L_{z} \sim 10^{3} \mathrm{~km}$ ).

The real heating time in this model is determined by the magnetic reconnection time scale, which is in the range 10$100 \mathrm{~s}$, and the volume heated by the waves is restricted in size to $\sim 10^{2}-10^{3} \mathrm{~km}$, equal to the dissipation distance of fast waves. A significant heating beyond this volume may be due to the thermal conduction from it.

\subsection{Nonlinear excitation of KAWs in the coronal holes}

The nonlinear properties of KAWs provide very promising plasma energization mechanisms that can be responsible for the plasma heating and solar wind acceleration in the coronal holes. We start from an initially static equilibrium where the plasma is heated locally, only at low heights (e.g., by acoustic shock waves), and the plasma temperature and density decrease with heliocentric distance along open magnetic field lines. The gas/magnetic pressure ratio $\beta$ should also decrease, practically to zero, at the distance of the order of hydrostatic scale of height. Let us now assume that a flux of MHD Alfvén waves is launched in the corona by photospheric motions (or by magnetic reconnection events at the coronal base). These waves propagate upward unattenuated up to the height where $\beta$ becomes equal to $m_{e} / m_{p}$. But, as soon as $\beta$ drops below $m_{e} / m_{p}$, MHD Alfvén waves undergo a strong parametric decay into KAWs. Due to their short perpendicular wavelengths, the nonlinearly excited KAWs dissipate via collisional or collisionless wave-particle interaction. This, in turn, gives rise to plasma heating and particles acceleration. Thus, the flux of KAWs, that propagates further upward, can easily increase $\beta$ again well above $m_{e} / m_{p}$ in the high corona and provide the energy source for the solar wind acceleration. This should eventually result in a new, dynamic equilibrium, in which (i) the first transition point from $\beta>m_{e} / m_{p}$ to $\beta<m_{e} / m_{p}$ is shifted to larger heights (result of the dissipation of downward KAW flux); (ii) there is an extended region where $\beta$ is kept (by the dynamical back reaction on the KAWs damping) at a level below $m_{e} / m_{p}$, so as to provide an efficient conversion of the upward MHD Alfvén flux into two counter-streaming KAW fluxes; (iii) there is a second transition point from $\beta<m_{e} / m_{p}$ to $\beta>m_{e} / m_{p}$, beyond which the plasma is strongly heated by the upward KAW flux and expands forming solar wind.

Let us turn to numerical estimations. We take values for plasma parameters that are typical for coronal holes. The temperatures and densities of electrons in coronal holes are rather well determined (see Wilhelm et al. (1998) and references therein). Near the critical point we take $\beta=$ $0.99 m_{e} / m_{p}, T_{e}=10^{5} \mathrm{~K}, n_{e}=3 \times 10^{7} \mathrm{~cm}^{-3}$, and $T_{p} / T_{e}=1.5, \Omega_{p}=5 \times 10^{4} \mathrm{~s}^{-1}$. The electron collisional frequency $v_{e}$ may then be estimated as $v_{e} \sim 100 \mathrm{~s}^{-1}$. As the collisional (resistive) dissipation of KAWs is stronger than the Landau damping for low-frequency waves $\omega_{k}<10 \mathrm{~s}^{-1}$ (Voitenko and Goossens, 2000), we use the collisional damping as an estimate of the dissipation rate:

$\gamma_{\mathrm{d} 1}=\gamma_{\mathrm{d} 2}=\gamma_{\mathrm{d}}=-0.25 v_{e} \frac{k_{\perp}^{2} \delta_{e}^{2}}{1+k_{\perp}^{2} \delta_{e}^{2}}$,

and

$\gamma_{\mathrm{tot}}=\gamma_{\mathrm{d}}+\gamma_{N L}$ 
The maximal growth $\gamma_{\text {tot }} \approx 0.6 \mathrm{~s}^{-1}$ is attained at $k_{\perp}^{2} \delta_{e}^{2} \approx 1$ if we take the (underestimated) amplitude $b_{\mathrm{P}}=0.0023$ of the MHD waves, participating in elementary three-wave interactions. Formally, we can have a much higher growth rate with different wave and plasma parameters, but we are restricted by the condition of weak growth/damping, i.e., $\gamma_{\text {tot }}$ should be smaller than $\omega_{\mathrm{P}}, \gamma_{\text {tot }}<\omega_{\mathrm{P}}$, which can be violated for the low-frequency MHD waves. To this end we note that the wave flux, coming from the $\beta>m_{e} / m_{p}$ region, meets initially very small values of $1-\beta m_{p} / m_{e}$, satisfying condition $\gamma_{\text {tot }}<\omega$, but providing fast enough conversion of wave energy. The mechanism is working in such a way as to keep $\beta$ very close to $m_{e} / m_{p}$, so that the values $\beta=0.99 m_{e} / m_{p}$ can be reasonable. But, for any $\beta<m_{e} / m_{p}$, the fastest growing waves have perpendicular wavenumbers in the range $k_{\perp}^{2} \delta_{e}^{2}=1-3$.

The condition for the non-adiabatic cross-field acceleration of oxygen ions $O^{+5}$ is

$\frac{m_{i}}{5 m_{p}} \frac{k_{x} V_{A}}{\Omega_{p}} \frac{1+\mu_{p}}{K} \frac{B_{y}}{B_{0}}>1$.

For the nonlinearly excited KAWs with $k_{x} \delta_{e} \gtrsim 1$ we obtain the following condition for relative KAW amplitudes

$$
\frac{B_{y}^{K A W}}{B_{0}}>\frac{5 m_{p}}{m_{i}} \sqrt{\frac{m_{e}}{m_{p}}} \frac{K}{k_{x} \delta_{e}\left(1+\mu_{p}\right)}=(1-3) \times 10^{-3} .
$$

Since the amplitudes of coronal MHD wave are in the range $B^{M H D} / B_{0}=0.05-0.1$, condition (48) becomes realistic even for a small fraction $(\lesssim 0.1)$ of MHD wave energy transferred to KAWs, and $O^{+\widetilde{5}}$ ions can be strongly heated in the perpendicular direction. However, due to the high efficiency of the nonlinear excitation of KAWs, we could expect much more energy to be transferred to KAWs, of the order of the initial MHD wave energy. In this case the protons can also be heated by KAWs. The condition for non-adiabatic proton acceleration is $B_{y}^{K A W} / B_{0} \gtrsim 0.01$. An essential feature of this heating mechanism is its relation to the region of $\beta \lesssim m_{e} / m_{p}$ at some distance from the Sun.

\subsection{Powering aurora}

A high level of KAWs is continuously observed by satellites in the auroral zones of terrestrial magnetosphere at heights $1.5 \div 6 \mathrm{R}_{E}$ ( $\mathrm{R}_{E}$ is Earth's radius), where these waves provide plasma heating and particle acceleration. Moreover, recent satellite observations indicate that the KAW flux is responsible for the most intense events observed in the aurora (Wygant et al., 2002; Chaston et al., 2003). However, the origin of these KAWs is still uncertain. At the same time satellites measure a large energy flux of large-scale MHD Alfvén waves that are excited by the solar wind interacting with the magnetosphere, and/or by the magnetic reconnection in the geomagnetic tail. These MHD waves propagate downward along geomagnetic field lines, and their flux is sufficient to power the measured flux of KAWs. This observational fact suggests (Wygant et al., 2002) that the KAWs are generated by MHD Alfvén waves. If we take the amplitude of the MHD Alfvén waves $b_{\mathrm{P}}=0.1$ and $\omega_{\mathrm{P}} / \Omega_{p}=0.01-0.1$ (Wygant et al., 2002), then we find from Eq. (39) a strong nonlinear pumping of energy into KAWs $\gamma_{N L \max } \sim \omega_{1} \lesssim \omega_{\mathrm{P}}$ in the region where $\beta_{m}$ is still very close to unity, $\beta_{m} \simeq 0.99$. For $\beta_{m} \lesssim 1$, the fastest growing KAWs have perpendicular wavenumbers $k_{\perp} \delta_{e} \gtrsim 1$. The Landau damping rate of these KAWs (Lysak and Lotko, 1996) is weaker than the nonlinear pumping rate, $\gamma_{L} \ll \omega_{1} \sim \gamma_{N L \max }$. Hence, the nonlinear excitation of KAWs by the decay of MHD Alfvén waves can be very efficient in the auroral regions.

The condition for non-adiabatic acceleration of $\mathrm{O}^{+}$ions can be written as

$\frac{k_{x} V_{A}}{\Omega_{p}}\left(\frac{1+\mu_{p}}{K}-\frac{V_{i z}}{V_{A}}\right) \frac{B_{y 0}}{B_{0}}>\frac{\Omega_{i}}{\Omega_{p}}=\frac{1}{16}$.

For KAW wave lengths of the order of electron inertial length, this gives very low critical amplitudes $B_{y 0} / B_{0}>$ $1.5 \times 10^{-3}$. KAWs with super-critical amplitudes are always observed in the auroral regions of strong cross-field heating of $\mathrm{O}^{+}$ions. These KAWs are accessible for the nonadiabatic acceleration of $\mathrm{O}^{+}$across the background magnetic field and are thus responsible for the observed heating and formation of ion conics.

\section{Conclusions}

We have studied the nonlinear excitation of KAWs by MHD Alfvén and fast waves. The parametric decay of a pump MHD Alfvén wave into KAWs is possible in a very low- $\beta$ plasma only, $\beta<m_{e} / m_{p}$. The pump fast magnetoacoustic wave can drive KAWs in a low- $\beta$ plasma, $\beta<1$. The main conclusion is that these elementary nonlinear processes can strongly accelerate the spectral evolution of wave energy from MHD length scales to dissipative short length scales in a variety of space plasmas.

The nonlinear excitation of KAWs in the extended solar corona can be provided by upward-propagating (Alfvén and fast) MHD waves launched from the convection zone or excited by the magnetic reconnection events at the coronal base. Short transversal wavelengths of the order $10 \mathrm{~m}$ make KAWs accessible for the non-adiabatic acceleration of oxygen ions if the KAW/background magnetic field ratio exceeds 0.005 . The resulting ion energization is primarily in the direction perpendicular to the background magnetic field. This process provides an alternative to the ion-cyclotron explanation for the intense transverse energization of oxygen ions observed by SOHO at 2-4 solar radii. Similarly, the impulsive heating events at the coronal base can be due to the dissipation of KAWs nonlinearly driven by MHD waves, excited by the collapsing current sheets.

In the terrestrial magnetosphere, a large flux of MHD Alfvén waves is generated by magnetic reconnection in the magnetotail and propagates downward. Under our hypothesis, this flux powers the energy release in the auroral zones by means of nonlinearly excited KAWs. The energy release is 
distributed between the field-aligned degree of freedom for the electrons, and the cross-field degree of freedom for the ions, as is observed by satellites.

Acknowledgements. This work was supported by the FWO Vlaanderen (research project G.0178.03) and by Onderzoeksfonds K. U. Leuven (research project OT/02/57).

Edited by: P.-L. Sulem

Reviewed by: R. A. Treumann and another referee

\section{References}

Berger, T. E., De Pontieu, B., Schrijver, C. J., and Title, A. M.: High-resolution Imaging of the Solar Chromosphere/Corona Transition Region, ApJ, 519, L97, 1999.

Berghmans, D., McKenzie, D., and Clette, F.: Active Region Transient Brightenings. A Simultaneous View by SXT, EIT and TRACE, A\&A, 369, 291, 2001.

Bhattacharjee, A., and Ng, C. S.: Random Scattering and Anisotropic Turbulence of Shear Alfvén Wave Packets, ApJ, 548, 318-322, 2001.

Chaston, C. C., Bonnell, J. W., Carlson, C. W., McFadden, J. P., Ergun, R. E., and Strangeway, R. J.: Properties of Small-scale Alfvén Waves and Accelerated Electrons From FAST, J. Geophys. Res., 108, A4, doi:10.1029/2002JA009420, 2003.

Cranmer S. R.: Coronal Holes and the High-Speed Solar Wind, Space Sci. Rev., 101, 229-294, 2002.

Dodero, M. A., Antonucci, E., Giordano, S., and Martin, R.: Solar Wind Velocity and Anisotropic Coronal Kinetic Temperature Measured with the O VI Doublet Ratio, Solar Phys., 183, 77-90, 1998.

Fushiki, T. and Sakai, J.-I.: Modulational Instability of Fast Magnetosonic Waves Emitted From a Pinching Current Sheet, Solar Phys., 150, 117-126, 1994.

Sagdeev, R. Z. and Galeev, A. A.: Nonlinear Plasma Theory, W. A. Benjamin, Inc., New York, 1969.

Hollweg, J. V.: Transition Region, Corona, and Solar Wind in Coronal Holes, J. Geophys. Res., 91, 4111-4125, 1986.

Hollweg, J. V. and Isenberg, P. A.: Generation of the Fast Solar Wind: A Review With Emphasis on the Resonant Cyclotron Interaction, J. Geophys. Res., 107, pp. SSH 12-1, doi:10.1029/2001JA000270, 2002

Kletzing, C. A., Bounds, S. R., Martin-Hiner, J., Gekelman, W., and Mitchell, C.: Measurements of the Shear Alfvén Wave Dispersion for Finite Perpendicular Wave Number, Phys. Rev. Lett., 90, id. 035004, 2003.

Kohl, J. L., Noci, G., Antonucci, E., et al.: First Results from the SOHO Ultraviolet Coronagraph Spectrometer, Solar Phys., 175, 613-644, 1997.

Leamon, R. J., Matthaeus W. H., Smith C. W., Zank G. P., and Mullan D.: MHD-driven Kinetic Dissipation in the Solar Wind and Corona, ApJ, 537, 1054-1062, 2000.

Leneman, D., Gekelman, W., and Maggs, J.: Laboratory Observations of Shear Alfvén Waves Launched from a Small Source, Phys. Rev, Letters, 82, 2673-2676, 1999.

Li, X., Habbal S.R., Hollweg J.V., and Esser, R.: Heating and Cooling of Protons by Turbulence-Driven ion Cyclotron Waves in the Fast Solar Wind, J. Geophys. Res., 104, 2521-2536, 1999.
Lysak, R. L. and Lotko, W.: On the Kinetic Dispersion Relation for Shear Alfvén Waves, J. Geophys. Res., 101, 5085-5094, 1996.

Markovskii, S. A. and Hollweg, J. V.: Parametric Cross-Field Current Instability in Solar Coronal Holes, J. Geophys. Res., 107, pp. SSH 21-1, doi:10.1029/2001JA009140, 2002.

Marsch E. and Tu C.Y.: Evidence for Pitch Angle Diffusion of Solar Wind Protons in Resonance With Cyclotron Waves, J. Geophys. Res., 106, 8357-8362, 2001.

Matthaeus, W. H., Mullan, D. J., Dmitruk, P., Milano, L. J., and Oughton, S.: MHD Turbulence and Heating of the Open FieldLine Solar Corona, Nonlin. Proc. Geophys., 10, 93-100, 2003, SRef-ID: 1607-7946/npg/2003-10-93.

Onishchenko, O. G., Pokhotelov, O. A., Sagdeev, R. Z., Stenflo, L., Treumann, R. A., and Balikhin, M. A.: Generation of Convective Cells by Kinetic Alfvén Waves in the Upper Ionosphere, J. Geophys. Res., 109, doi:10.1029/2003JA010248, 2004.

Pokhotelov, O. A., Onishchenko, O. G., Sagdeev, R. Z., and Treumann, R. A.: Nonlinear Dynamics of Inertial Alfvén Waves in the Upper Ionosphere: Parametric Generation of Electrostatic Convective Cells, J. Geophys. Res., 108, pp. SMP 15-1, doi:10.1029/2003JA009888, 2003.

Ryutova, M., Habbal, S., Woo, R., and Tarbell, T.: Photospheric Network as the Energy Source for the quiet-Sun corona, Solar Phys., 200, 213-234, 2001.

Sakai, J.-I.: Modulational Instability of Fast Magnetosonic Waves in a Solar Plasma, Solar Phys., 84, 109-118, 1983.

Stasiewicz, K., Bellan, P., Chaston, C., et al.: Small Scale Alfvénic Structure in the Aurora, Space Sci. Rev., 92, 423-533, 2000.

Stasiewicz, K., Khotyaintsev, Y., and Grzesiak, M.: Dispersive Alfvén Waves Observed by Cluster at the Magnetopause, Physica Scripta, T107, 171-179, 2004.

Verheest, F.: Alfvén Instabilities in Streaming Plasmas With Anisotropic Pressures and Their Relevance for the Solar Wind, Astrophys. Space Sci., 46, 165-173, 1977.

Voitenko, Yu. M.: Three-wave Coupling and Parametric Decay of Kinetic Alfvén Waves, J. Plasma Phys., 60, 497-514, 1998.

Voitenko, Yu. M.: Three-wave Coupling and Weak Turbulence of Kinetic Alfvén Waves, J. Plasma Phys., 60, 515-527, 1998.

Voitenko, Yu. and Goossens, M.: Nonlinear Decay of Phase-Mixed Alfvén Waves in the Solar Corona, A\&A, 357, 1073-1085, 2000.

Voitenko Yu. M. and Goossens M.: Excitation of High-Frequency Alfvén Waves by Plasma Outflows From Coronal Reconnection Events, Solar Phys., 206, 285-313, 2002a.

Voitenko, Yu. M. and Goossens, M.: Nonlinear Excitation of SmallScale Alfvén Waves by Fast Waves and Plasma Heating in the Solar Atmosphere, Solar Phys., 209, 37-60, 2002b.

Voitenko, Yu. M. and Goossens, M.: Cross-Field Heating of Coronal Ions by Low-Frequency Kinetic Alfvén Waves, ApJ, 605, L149-L152, 2004.

Wilhelm K., Marsch E., Dwivedi B. N., et al.: The Solar Corona above Polar Coronal Holes as Seen by SUMER on SOHO, ApJ, 500, 1023-1038, 1998.

Wygant, J. R., Keiling, A., Cattell, C. A., et al.: Evidence for Kinetic Alfvén Waves and Parallel Electron Energization at 4-6 RE Altitudes in the Plasma Sheet Boundary Layer, J. Geophys. Res., 107, pp. SMP 24-1, doi:10.1029/2001JA900113, 2002. 